\title{
Damage identification method of suspension bridge structure based on wavelet - artificial immune algorithm
}

\author{
Deqing Guan ${ }^{1, a^{*}}$, Deng Luo ${ }^{2, b}$ and Ran $\mathrm{Li}^{3, \mathrm{c}}$ \\ ${ }^{1,2,3}$ School of Civil Engineering and Architecture,Changsha University of Science and \\ Technology,Changsha 410114, china \\ a*491596452@qq.com, bluodeng110@163.com, ${ }^{\mathrm{c}} 421359528 @ q q . c o m$
}

\begin{abstract}
Keywords: Damage, Wavelet analysis, Artificial immune algorithm, Suspension bridge structure Abstract. Structural damage detection and diagnosis is a very important factor to the structural safety. Wavelet transform combined with artificial immune algorithm can be used for improving the application of artificial intelligence algorithms.for structural damage detection. The result of the simulation and verification by analyzing suspension bridge structure suggest that this approach combines the advantages of wavelet analysis and artificial immune algorithm : quickly and accurately identifying the damage location and calculating the extent of the damage. This method can be used in engineering practice of structural damage detection to improve the efficiency of structural damage identification.
\end{abstract}

\section{Introduction}

In recent years, with the rapid development in the field of civil engineering structures, all kinds of complicated structures appear constantly, and the old structure will be affected by all kinds of load and sudden external factors in the long-term complex service environment which will inevitably lead to structure damage accumulation and resistance attenuation of the system, and in extreme cases this could lead to catastrophic accidents. Therefore how to judge whether there is damage and where the damage location is and evaluation through certain means of detection and the method have become the concerned and active field of research by domestic and international academia and engineering . In 1974,the concept of wavelet transform was first proposed by the engineer J.Morlet who was engaged in signal processing of oil in France. Then ,it's gradually formed with the development of junior scientists Y.M eyer, S.M allat, I.Daubechies and etc. Hong [1] found that based on the wavelet singularity analysis for a beams of crack damage detection, through wavelet transform beam's first-order modal vibration mode for continuous wavelet analysis, using wavelet transform modulus maxima point position can accurately determine the position of the beam damage.Shi [2] use the curvature modal of the continuous steel box girder to make a damage identification on a cracks of continuous steel box girder. Douka [3] use damage cantilever beams with the wavelet transform and intensity factor to determine the damage location and damage degree, the numerical analysis show that the damage factor has high accuracy in determining the damage position. M.Rucka ect [4] use one dimensional continuous wavelet to analyse a cantilever beam with damage model and use two-dimensional wavelet to analyse four edges fixed plate model of wavelet. The results show that the corresponding damage location where the wavelet mathematical model arrive at the maximum point and where it insured by the wavelet analysis method are not dependent on the characteristics of the structure and mathematical model.Deqing Guan etc [5] put forward that the problem of frame structure damage identification of strain mode can be solved by using wavelet transform. The results show that the method is able to identify damage of frame structure. Deqing Guan etc [6] put up with using avelet theory to solve such as elastic plate damage identification, the numerical analysis shows that the method is effective and accurate, and has some practical value in engineering. Wavelet analysis is the perfect combination of mathematics and engineering technology, the rapid development of it and gradually perfect provide a very reliable data processing method for structural damage diagnosis, especially has obvious advantages in the analysis of structural damage location . 
Artificial immune algorithm is a kind of new type of intelligent algorithm in the structural damage research. Researchers apply concept and theory of immune in the genetic algorithm ,under the premise of keeping the good qualities of original algorithm, They try to use some characteristics of the information or knowledge of the unsettled problems to suppress the degradation phenomenon appeared in the process of optimization, the algorithm is called immune algorithm [7-8]. Based on characteristics of antigen and antibody combined with the each other, A Tarakanov [9] established a systematic model of artificial immune system, and points out that the model is used for evaluation of kaliningrad ecology atlas complex calculation safter improvement. J Timmis [10] put forward a kind of artificial immune system approach which can resource constraints, the algorithm is based on natural population control mechanism of the immune system, control of population growth and conditions for termination of algorithm, and successfully used in fisher petals problems. Nohara [11] put forward a kind of network model of artificial immune system based on the function of antibody unit. Wanquan Sun etc [12] apply the immune algorithm to structure dynamic parameter recognition and it is identified that immune algorithm can realize the structural dynamic parameters quickly and accurately and has better ability to resist noise. Michelle etc [13] has studied the bionic mechanism of the artificial immune system and put forward a artificial immune model based on the diagonal distance to solve the problem of structural damage identification and classification, and has obtained certain achievement. Studies show that the artificial immune algorithm is better than traditional artificial intelligence algorithm in stronger ability of global optimization, faster convergence speed and better robustness, when applied to the structural damage diagnosis, it will have a very broad prospects.

\section{The principle of wavelets-artificial immune algorithm in damage identification}

\section{Damage location identification of structure based on continuous wavelet transform}

Assuming that the function satisfies the following conditions:

$$
\begin{aligned}
& C_{\psi}=\int_{-\infty}^{+\infty} \frac{|\psi(w)|^{2}}{w} d_{w}, \psi_{a, \tau}(t)=\frac{1}{\sqrt{2}} \psi\left[\frac{t-\tau}{a}\right], \psi(t) \in L^{2}(R) . \\
& W f(a, \tau)=\frac{1}{\sqrt{a}} \int_{-\infty}^{+\infty} f(t) \psi^{*}\left[\frac{t-\tau}{a}\right] d t .
\end{aligned}
$$

Where $\psi(\mathrm{t})$ is the mother wavelets, $\psi(\omega)$ is the form of fourier transformation of $\psi(\mathrm{t}) . \psi(\omega)$ can be obtained by translated as well as lengthened or shortened of $\psi(t)$. a is the scale factor and $\tau$ is the shift factor. Where $\psi^{*}(\mathrm{t})$ is the conjugate function of $\psi(\mathrm{t})$.

According to the equation (2), local features of the function can be highlighted by the continuous wavelets transformation both in time domain and in frequency domain. Then according to the singularity of the coefficient diagram get the damage location of structure.

The calculating principle of damage degree identification based on the artificial immune algorithm

Artificial immune algorithm is a learning and optimization algorithm which based on biological immune system and learn from its running mechanism. So,the artificial immune algorithm has excellent global search ability for the degree of structural damage identification. The antigens and antibodies of artificial immune algorithm, respectively, corresponding to the objective function and feasible solutions of optimization problems. In this paper, using the matlab language to compile artificial immune procedure to identify structural damage degree,the process includes: (1) Encoding (2) Estabilish the objective function and determine the population scale (3) Affinity calculation (4) The similarity of antibodies (5)

The discriminant of termination conditions (6) Antibody concentration (7) The antibody promotion and suppression and crossover andvariation.

The objective function of the program is founded based on the structure frequency and modal shape. Using the error function of structure frequency and modal shape, the calculation equation can be expressed as following equation: 


$$
J=F_{\omega} \sum_{i=1}^{m}\left(\frac{f_{i}^{\text {test }}-f_{i}^{c a l}}{f_{i}^{\text {test }}}\right)^{2}+F_{\varphi} \sum_{i=1}^{n} \sum_{j=1}^{k}\left(\varphi_{i j}^{\text {test }}-\varphi_{i j}^{\text {cal }}\right)^{2} .
$$

$F_{\omega}$ and $F_{\varphi}$ are weighted factors, $f_{i}^{t e s t}$ is the vibration frequency of field measurement and $f_{i}^{c a l}$ is the vibration frequency of calculation. $\varphi_{i j}^{\text {test }}$ and $\varphi_{i j}^{\text {cal }}$,respectively, are the vibration frequency of field measurement and calculation which are normalized.m is the frequency order and $n$ is the vibrational order of the calculation and evaluation and $\mathrm{k}$ is the number of mode displacement. $\mathrm{J}$ is smaller the value and the better the calculation result matches with actual damage degree.

\section{Numerical simulating analysis}

This paper use the following model and proposed condition to test the validity of the damage identification method for structure as previously described.The paper establish three-span suspension bridge structure as shown in Fig.1.Overview: material is steel, midspan is 120 meters, both side spans are 60meters.the main bean cross-sectional dimensions: $\mathrm{b} \times h=3500 \mathrm{~mm} \times 2500 \mathrm{~mm}$, elastic modulus $E=3.0 \times 10^{11} \mathrm{~N} / \mathrm{m}^{2}$, density $\rho=10000 \mathrm{~kg} / \mathrm{m}^{3}$, poisson's ratio $\mu=0.3$. the suspension bridge is divided into 40 units equidistant by the finite element method.this article assumes that:the 10th and 20th have damage, respectively,are $20 \%$ and 25\%.Damage identification of the suspension bridge structure based on continuous wavelet transform of rotation mode select $\mathrm{db}$ wavelet as the mother wavelet and the wavelet coefficient output as shown in Fig.2.Absolutely,the 10th and 20th units are damage location.Similarly,load the damage location into the wavelet-artificial immune algorithm program, then the wavelet-artificial immune algorithm program could analyze and calculate the degree of damage to the two injuries of outcome as shown in Fig.3.

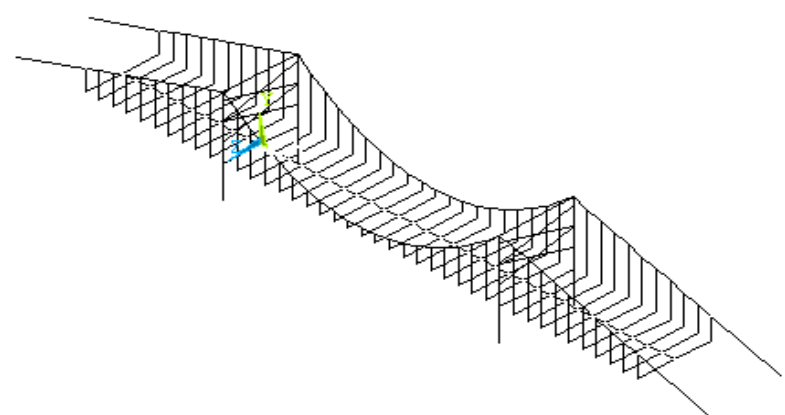

Fig.1. suspension bridge structure

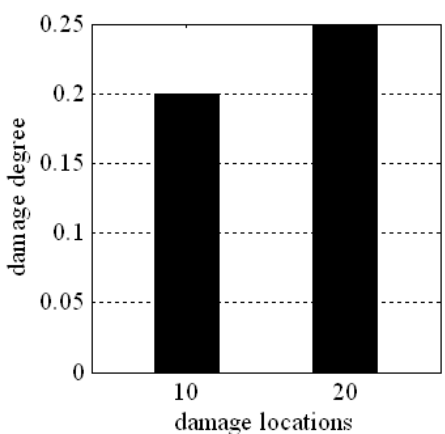

Fig.3. damage identification results

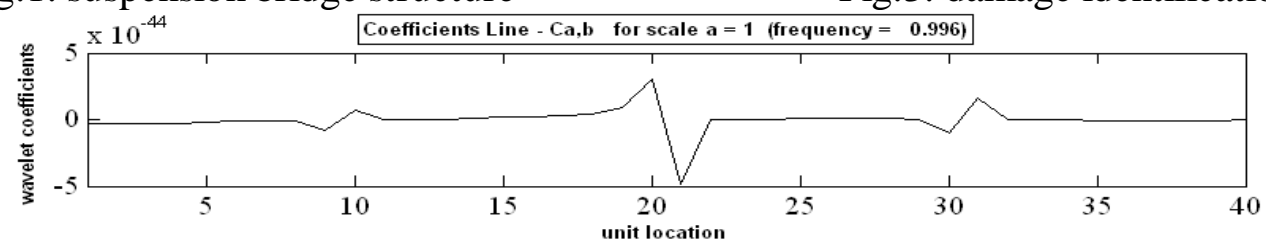

Fig.2. wavele coefficients of suspension bridge structure with two damages

On the premise of the wavelet localization, the degree of the damage can be precisely geted in the preset value: in Fig.3, the damage degree of unit 10 is $20 \%$, the damage degree of unit 20 is $25 \%$. The results are consistent with the answers set.

\section{Conclusions}

This is the first time to combine wavelet transform and artificial immune algorithm for analysis and calculation of the structure damage diagnosis and this new method can be called the wavelet-artificial immune algorithm. This paper proved that damage identification of the suspension bridge structure could be valid diagnosed.

It can be seen, the damage location has indeed reached a certain degree of precision in the wavelet coefficient table and the damage degree of every damage locations are also in accordance with the 
answers preseted. So the wavelet-artificial immune algorithm not only can be identified the damage location effectively,but also calculated the damage degree accurately.

In this paper, wavelet transform would be used determining the damage location. Assumption,the unknown numbers of artificial immune algorithm are greatly reduced while calculating the damage degree.The wavelet-artificial immune algorithm learned the advantages of wavelet transform and artificial immune algorithm:precise identification and accurate calculation.

So long as puted the modal parameters into the compiled wavelet-artificial immune algorithm program, damage location and damage degree of the suspension bridge structure can be identified by the program. The operation is simple and effective and this new method is convenient and accurate and have good development prospects. Actually this paper is only applied to suspension bridge structures, but there is no doubt that it could be applied to other complicated structures in the future.

\section{Acknowledgement}

This work was funded by National Natural Science Foundation Project of China (No.51378079).

\section{References}

[1]Hong J C,Kim Y Y,Lee H C,et al.Damage detection using the Lipschitz exponent estimated by the wacelet transform applications to vibration modes of a beam,International Journal of Solids and Structures. 39 (2002) 1803-1816.

[2]Shi L C,Guan D Q,Jiang Z K,Research on damage of continuous steel girders identification by wavelet analysis of the curvature mode,Proceedings of Sixth International Confernce on Advances in Steel Structures,Hongkong:ICASS.(2009) 201-109.

[3]E Douka, S Loutridis, A Trochidis. Crack identification in beams using wavelet analysis, International Journal of Solids and Structurcs. 40(6) (2003) 3557-3596.

[4]M Rucka,K Wilde. Application of continuous wavelet transform in vibration based damage detection method for beams and plates,Journal of Sound and Vibration. 297(3-5) (2006) 536-550.

[5] Deqing Guan, Xiaolin Zhong, Hongwei Ying. Research on sheet crack identification of frame structure using wavelet analysis. Applied Mechanics and Materials. 71-78 (2011) 4074-4077.

[6] Deqing Guan, Hongwei Ying , Xiaolin Zhong. Damage identification of elastic plate based on strain mode wavelet analysis. Applied Mechanics and Materials. 71-78 (2011) 4078-4081.

[7]J. D. Farmer, N. H. Packard and A. S. Perelson. The immune system.Adaptation and Machine Learning. Physics 22D, (1987)

[8]H. Bersini and F. J. Varela.Hints for adaptive problem solving gleaned from immune networks. Proceedings of the first workshop on parallel problem solvingi from nature. Springier \& Verlagi, (1990)

[9]A Tarakanov, D Dasgupta. A formal model of an artificial immune system,BioSystems.55 (2000) $151-158$.

[10]J Timmis, M Neah, J Hunt. Data analysis using artificial immune systems, cluster analysis and Kohonen networks:some comparisons, IEEE SMC'99 Conference Proceedings, Institute of Electrical and Electronics Engineers, Incorporated.3 (1999) 922-927.

[11]B T Nohara, H Takahashi . Evolutionary computation in engineering artificially immune (EAI) system, IECON 2000 26th Annual Confjerence of the IEEE Industrial Electronics Society,US: IEE

Press.4 (2000) 2501-2506.

[12] Wanquan Sun, Zhenyue MA. Identification of structure dynamic parameter based on an immune algorithm , Chinese Journal of Computational Mechanics. 22(2) (2005) 155-159. (in Chinese)

[13] Yue Zhou, Shi Tang, Xuesong Jia. Structural Damage Detection and Classification Algorithm Based on Artificial Immune Pattern Recognition, Journal of Shenyang Jianzhu University(Natural Science). 29(2) (2013) 378-384.(in Chinese) 